\title{
Preparation of Polythiophene Films Showing Optical Activity by Electrochemical Polymerisation in Cholesteric Liquid Crystal Containing Coumarine
}

\author{
Hiroki Hayashi, Hiromasa Goto* \\ Division of Materials Science, Faculty of Pure and Applied Sciences, University of Tsukuba, \\ Tsukuba, Ibaraki 305-8573, Japan \\ *E-mail address: gotoh@ims.tsukuba.ac.jp
}

\begin{abstract}
We carried out electrochemical polymerisation in a cholesteric liquid crystal electrolyte solution. The polymer film prepared in the cholesteric liquid crystal showed chiropticality even though its monomer is an achiral. The surface morphology of the polymer was observed with polarising optical microscopy. Optical and electric properties were examined by UV-vis optical absorption, circular dichroism spectroscopy, and cyclic voltammetry.
\end{abstract}

Keywords: chiral inducer; coumarine; electrochemical polymerisation; liquid crystals

\section{INTRODUCTION}

Electrochemical polymerisation is one of the effective methods for preparation of conjugated polymer films. Mechanism of the polymerisation is considered to be oxidative reaction.

In general, common solvents such as acetonitrile, sulfuric acid aqueous solution, and propylene carbonate have been employed for electrochemical polymerisation [1-3]. Although these isotropic liquids are suitable for electrochemical polymerisation, control of the conformation and morphology of the polymer chains cannot be performed.

Recently, electrochemical polymerisation in liquid crystal has been developed, and the resultant polymer films have the similar form to that of the matrix liquid crystal [4-5].

In this study, we carried out electrochemical polymerisation of an achiral monomer in cholesteric liquid crystal medium containing coumarine. The polymer film shows optical activity, although the monomer is achiral. This indicates cholesteric liquid crystal as chiral solvent for electro-polymerisation can induce chirality of the polymer film.

\section{EXPERIMENT}

\section{1. Synthesis of chiral inducer}

1,10-di[[(S)-1-methyl-heptyl]biphenyl-4-carboxylate-4'-oxy]decane $(2 S)$ was prepared by the previously reported method [6]. 
Firstly, 4-hydroxybiphenyl-4'-carboxylic acid was coupled with an opticallyt active octanol $((R)$-2-octanol) with the Mitsunobu reaction. The resultant material was dimerisation via Williamson etherification reaction to obtain compound $2 S$, as shown in Scheme 1.

\section{4'-Hydroxybiphenyl-4-carboxylic Acid (S)-1-methyl-heptyl ester (1S)}

To a solution of diethylazodicarboxylate $(0.9 \mathrm{~mL}, 2.3 \mathrm{mmol})$ and 4-hydroxybiphenyl-4'carboxylic acid (499 mg, $2.3 \mathrm{mmol}$ ) in tetrahydrofuran (THF, $10 \mathrm{~mL}$ ) were added a solution of triphenylphosphine $(606 \mathrm{mg}, 2.3 \mathrm{mmol})$ and $(R)$-2-octanol $(387 \mathrm{mg}, 2.9 \mathrm{mmol})$ in THF (90 $\mathrm{mL}$ ). After stirring for $24 \mathrm{~h}$ at room temperature, the solvent was evaporated. The crude product was purified by silica gel chromatography (eluent: chloroform/ethyl acetate $=6 / 1$ ) to afford white solid (274 mg, 36\%).

\section{1,10-di[[(S)-1-Methyl-heptyl]biphenyl-4-carboxylate-4'-oxy]decane (2S)}

A solution of 4'-hydroxybiphenyl-4-carboxylic acid (S)-1-methyl-heptyl ester (1S) (250 $\mathrm{mg}, 0.77 \mathrm{mmol}$ ), 1,10-dibromodecane (118 mg, $0.38 \mathrm{mmol}$ ), and $\mathrm{K}_{2} \mathrm{CO}_{3}$ (424 mg, $3.06 \mathrm{mmol}$ ) in $N, N$-dimethylformamide $(10 \mathrm{~mL})$ was stirred for $24 \mathrm{~h}$ at $90{ }^{\circ} \mathrm{C}$. The reaction mixture was extracted with ethyl acetate 3 times. The organic layer was dried over magnesium sulfate and purified by silica gel chromatography (eluent: $n$-hexane/ethyl acetate $=9 / 1$ ). The solvent was removed under vacuum to afford yellow solid $(8.8 \mathrm{mg}, 1.4 \%)$.

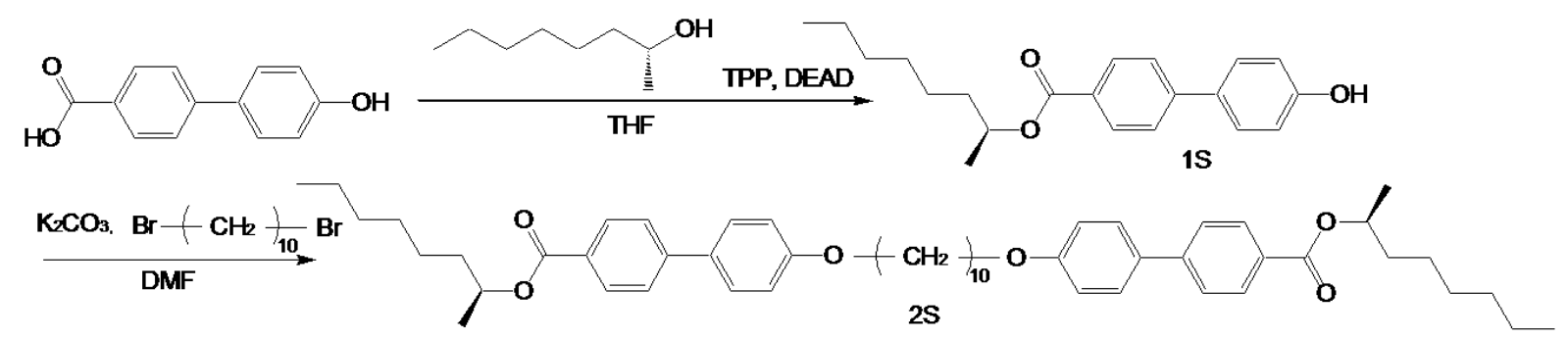

Scheme 1. Synthesis of chiral inducer. TPP = triphephylphosphine, DEAD = diethyl azocarboxylate, $\mathrm{THF}=$ tetrahydrofuran, $\mathrm{DMF}=N, N$-dimethylformamide.

\section{2. Preparation of cholesteric electrolyte solution and polymerisation in cholesteric liquid crystal}

Cholesteric liquid crystal materials can be obtained by adding chiral inducer to nematic liquid crystal. $n$-Hexylcyanobiphenyl (6CB, nematic liquid crystal) and an addition of small amount of chiral inducer $2 S$ induces cholesteric liquid crystal. Figure 1 (left) shows polarising optical microscopy image of the cholesteric electrolyte solution containing $6 \mathrm{CB}$, the monomers, and $n$-tetrabutyl ammonium perchlorate (TBAP). Quantity used; $2 S$ (chiral inducer, $2.96 \mathrm{mg}$ ), TBAP (supporting salt, $0.511 \mathrm{mg}$ ), terthiophene (monomer, $1.67 \mathrm{mg}$ ), coumarine (additive, $1.43 \mathrm{mg}$ ) 6CB (nematic liquid crystal, $89.04 \mathrm{mg}$ ). This is so called fingerprint texture, which is a typical of cholesteric liquid crystal.

Electochemical polymerisation in cholesteric liquid crystals was carried out by applying voltage of $4 \mathrm{~V}$ across indium-tin-oxide (ITO)coated glass cell charged with the cholesteric electrolyte solution containing the monomers (terthiophene) with $0.2 \mathrm{~mm}$ Tefron spacer (Scheme 2). After application of voltage for $30 \mathrm{~min}$, the resultant polymer film was washed with hexane and tetrahydrofuran to remove the liquid crytal electrolyte solution. Figure 1 
(right) shows polarising optical microscopy image of the polymer film. The texture is not similar to that of cholesteric liquid crystal but some morphology may be transcribed from the liquid crystalline medium.<smiles>CC(C)(C)c1ccc(-c2ccc(-c3ccc(-c4ccc(C(C)(C)C)s4)s3)s2)s1</smiles>

Scheme 2. Electrochemical polymerisation. $\mathrm{DC}=$ direct current.

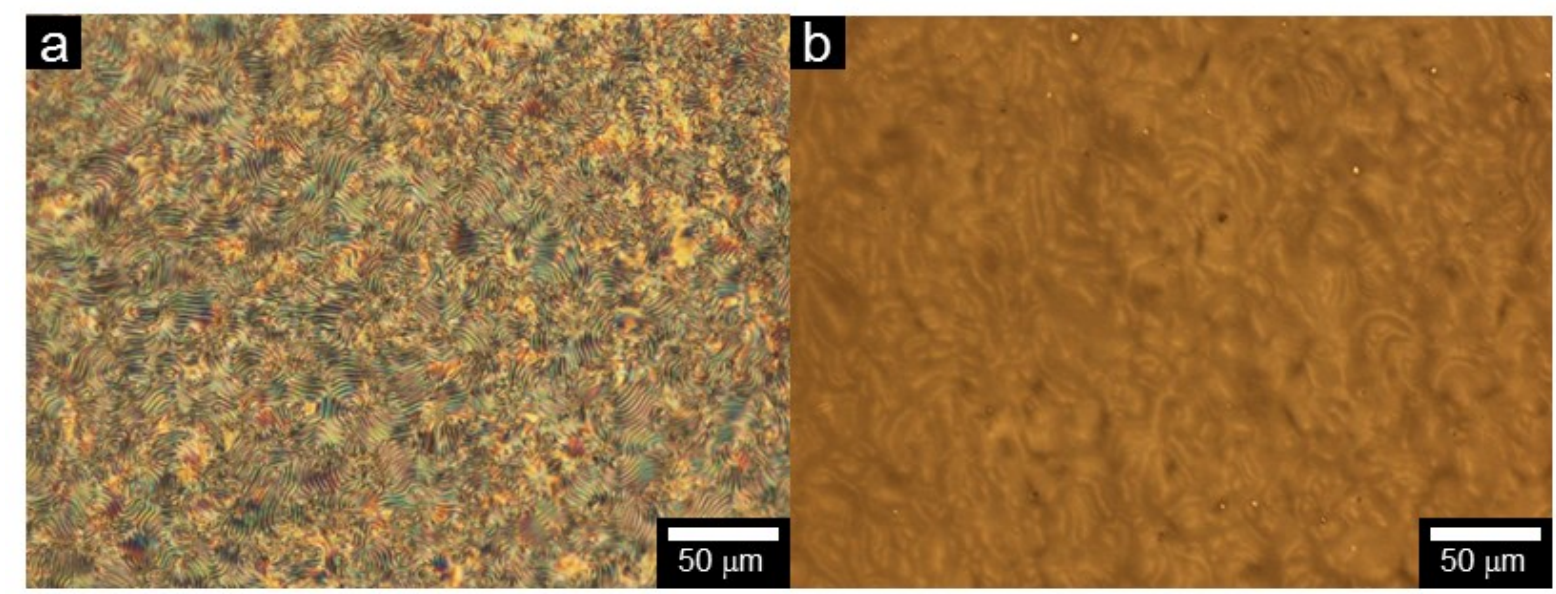

Figure 1. Polarising optical microscopy image of the cholesteric electrolyte solution (left, a) and the resultant polymer (right, b).

\section{3. Infrared absorption spectroscopy}

The polymer, coumarine and chiral inducer $(2 \mathrm{~S})$ were characterized by infrared absorption spectroscopy (Figure 2). The polymer does not show the signal at around 1710 $\mathrm{cm}^{-1}$, which is characteristic of $\mathrm{C}=\mathrm{O}$ vibration.

This suggests that the coumarine molecules and chiral inducer $(2 \mathrm{~S})$ are nocaptured into the polymer. 


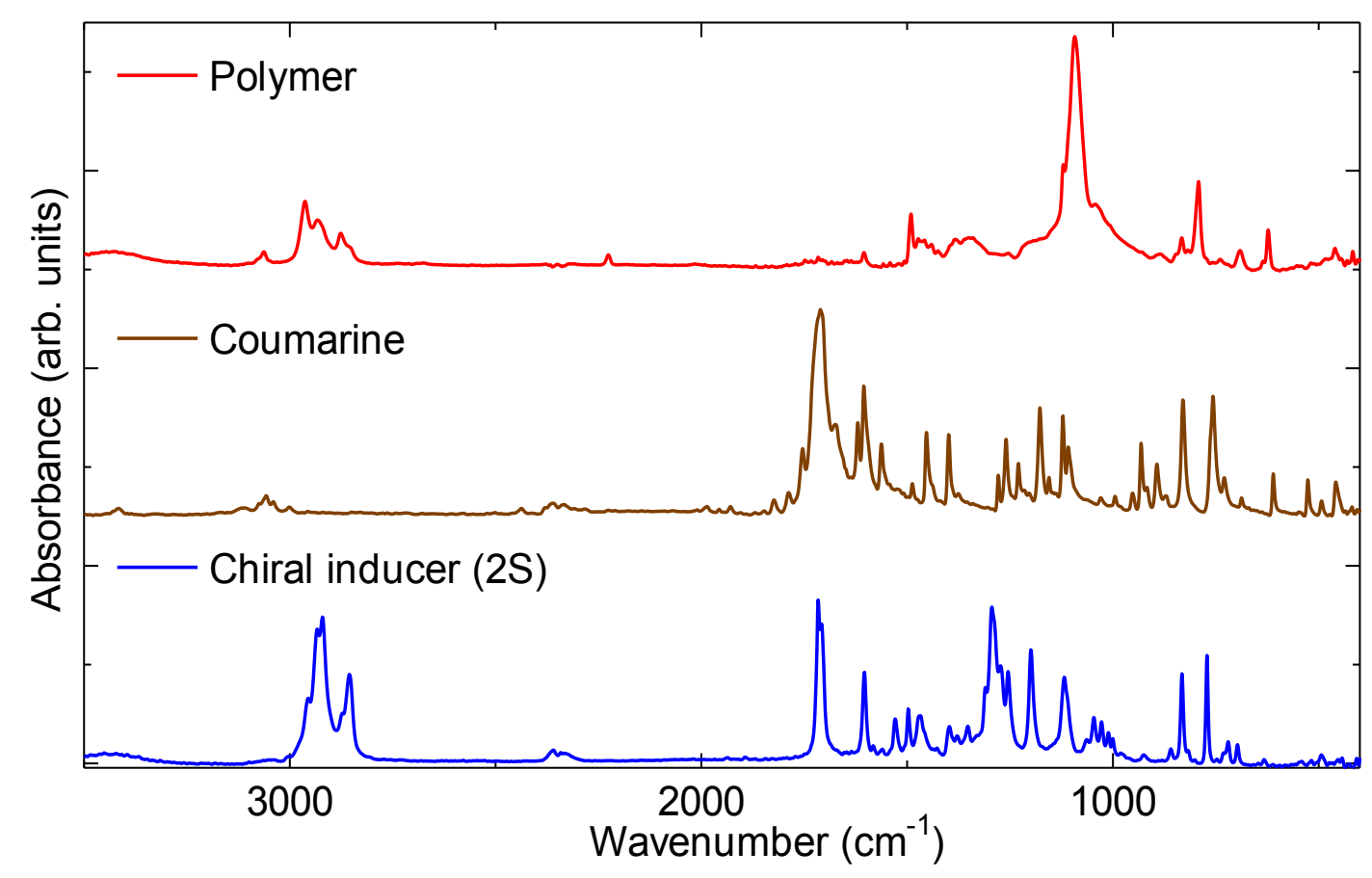

Figure 2. Infrared absorption spectra of the polymer film, coumarine, and the chiral inducer.

\section{4. UV-vis absorption spectroscopy}

Figure 3 shows absorption spectrum of the polymer film in the reduced state. The maximum absorption wavelength is at around $450 \mathrm{~nm}$ due to $\pi-\pi^{*}$ transition of the main chain.

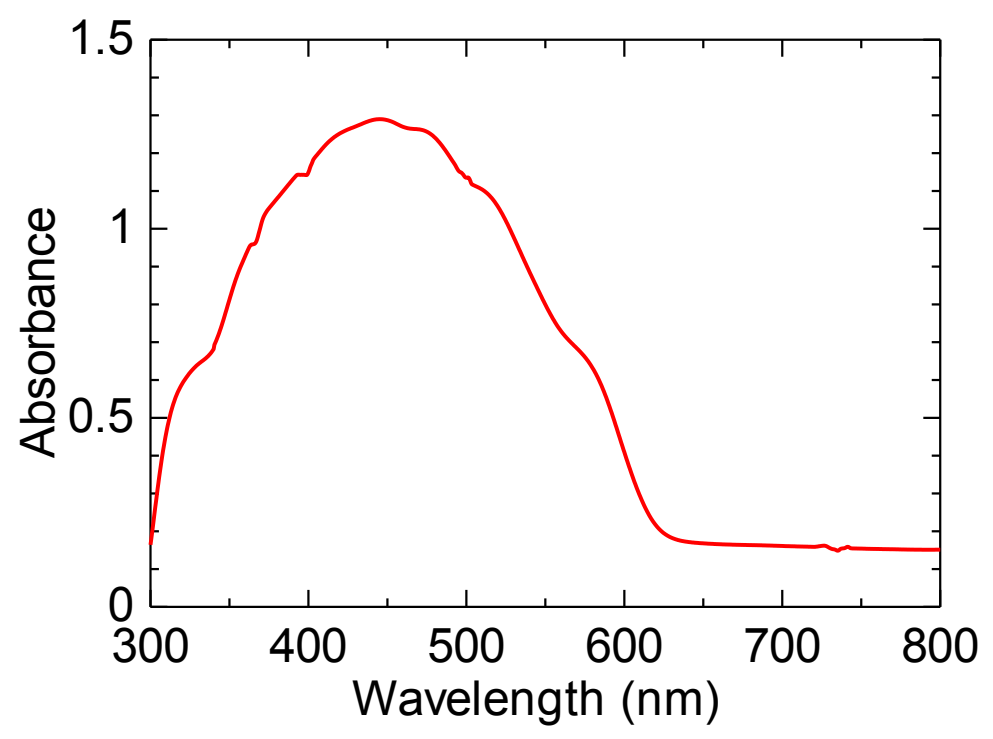

Figure 3. UV-vis absorption spectrum of the polymer film. 


\section{5. Circular dichroism spectroscopy}

Figure 4 displays circular dichroism (CD) spectrum of the polymer film in the reduced state, showing first-negative and second-positive Cotton effect from $600 \mathrm{~nm}$ to $300 \mathrm{~nm}$. In addition, the transition point from negative to positive corresponds to the maximum absorption wavelength $(450 \mathrm{~nm})$. This type of Cotton effect is referred to as Davydov splitting, indicating the polymer forms left handed helical aggregation. [7]

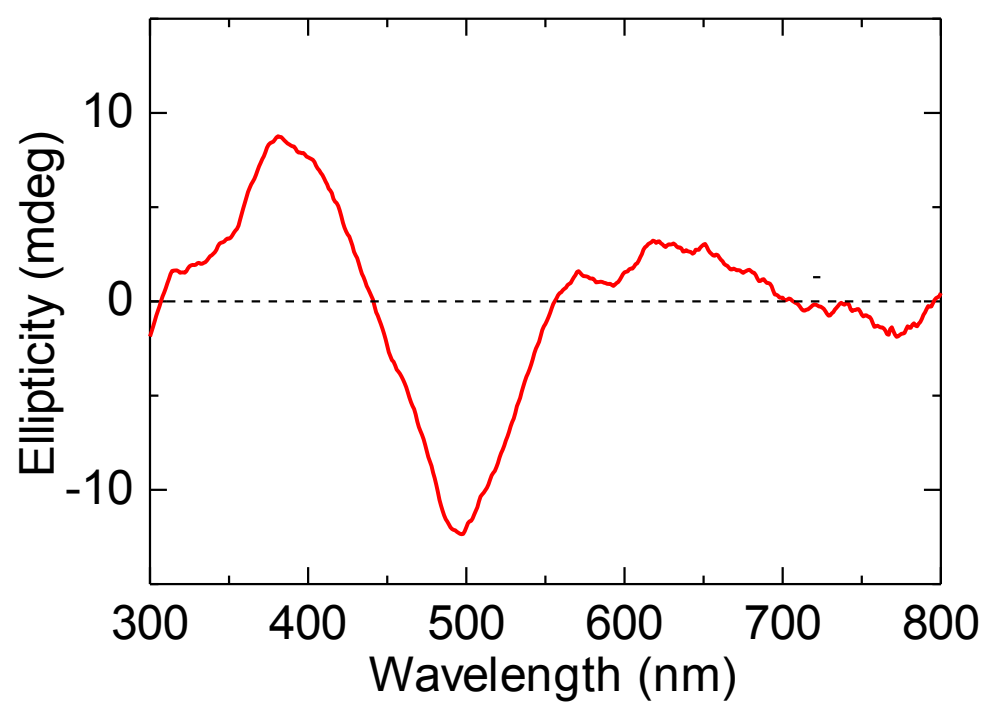

Figure 4. Circular dichroism spectrum of the polymer film.

\section{6. Cyclic voltammetry}

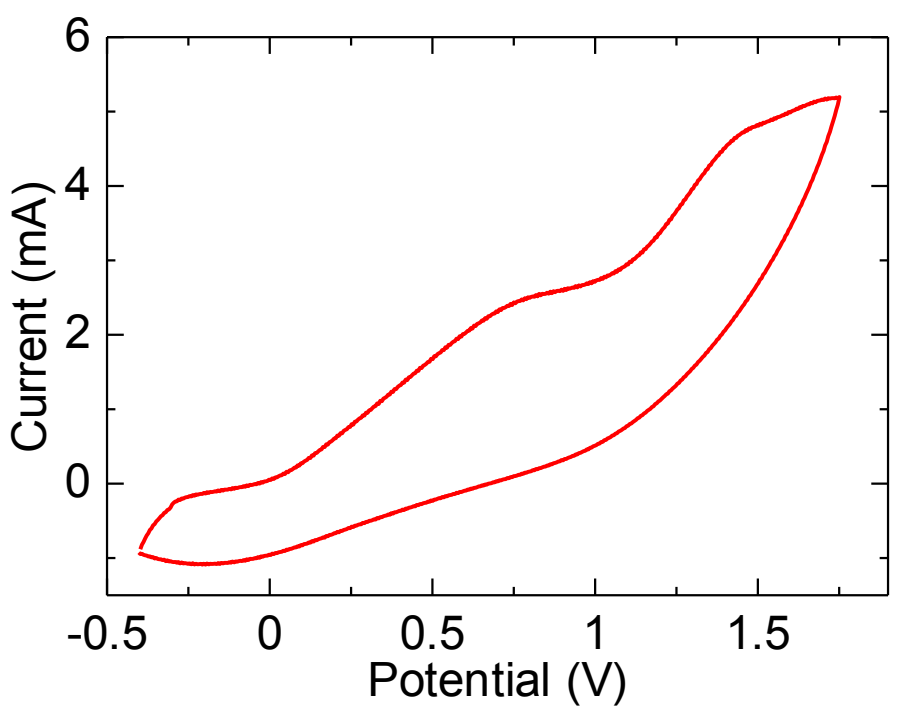

Figure 5. Cyclic voltammogram of the polymer film. 
Figure 5 shows the cyclic voltammetry of the polymer film on an ITO working electrode versus $\mathrm{Ag} / \mathrm{Ag}^{+}$reference electrode with a Pt wire counter electrode immersed in 0.1 M TBAP in acetnitrile at a rate of $100 \mathrm{mVs}^{-1}$. This redox behavior is a typical of conducting polymers. On the oxidation process, the peaks around 0.75 and $1.4 \mathrm{~V}$ are attributed to the generation of polarons and bipolarons, respectively.

\section{CONCLUSIONS}

Electrochemical polymerisation in cholesteric liquid crystal produced the polymer film. The polymer film shows optical activity even though the surface morphology shows no fingerprint texture. This indicates that cholesteric liquid crystal medium induces chirality to the polymer, and cholesteric liquid crystal like fingerprint texture is not essentially required for optical activities. Moreover, if coumarine molecules are captured into polymers, this composite would exhibit fluorescence with electro- chiroptical activity.

\section{Acknowledgments}

NMR measurements were carried out in Research Facility Center for Science and Technology University of Tsukuba.

\section{References}

[1] B. Fabre, J. C. Clark, M. Graça, H. Vicente, Macromolecules 39 (2006) 112-119.

[2] A. Watanabe, K. Mori, A. Iwabuchi, Y. Iwasaki, Y. Nakamura, O. Ito, Macromolecules 22 (1989) 3521-3525.

[3] R. J. Willicut, R. L. McCarley, J. Am. Chem. Soc. 116 (1994) 10823-10824.

[4] S. Ohkawa, R. Ohta, K. Kawabata, H. Goto, Polymers 2 (2010) 393-406.

[5] H. Goto, Macromolecules 40 (2007) 1377-1385.

[6] H. Hayashi, K. Kawabata, H. Goto, International Letters of Chemistry, Physics and Astronomy 8 (2014) 6-12.

[7] N. Harada, K. Nakanishi, J. Am. Chem. Soc. 91 (1969) 3989. 